\title{
Transitivity of interval and fuzzy-interval extensions of interval functions
}

\author{
H. Román-Flores ${ }^{1}$ Y. Chalco-Cano ${ }^{1}$ \\ ${ }^{1}$ Instituto de Alta Investigación, Universidad de Tarapacá, Casilla 7D, Arica, Chile
}

\begin{abstract}
Let $I=[a, b]$ be a real compact interval and $f: I \rightarrow$ $I$ a continuous function. Define $\mathcal{K}_{c}([a, b])$ the class of all non empty compact subinterval of $[a, b]$ and let $\bar{f}$ the natural extension of $f$ to $\mathcal{K}_{c}([a, b])$, that is to say, $\bar{f}(J)=f(J)$ for all $J \in \mathbb{I}([a, b])$. Also, let $\mathcal{F}_{c}([a, b])$ the class of all fuzzy-intervals with support contained in $[a, b]$ and consider $\hat{f}$ the Zadeh's extension of $f$ to $\mathcal{F}_{c}([a, b])$.

The aim of this paper is to show that $\bar{f}$ and $\hat{f}$ are not transitive for any arbitrary self continuous function $f$ defined on $I$.
\end{abstract}

Keywords: Transitivity, Zadeh's extensions

\section{Preliminaries and basic results}

The following diagram

$$
\begin{array}{lll}
(\mathcal{K}(X), H) & \stackrel{\bar{f}}{\longrightarrow} & (\mathcal{K}(X), H) \\
\uparrow & \uparrow \\
(X, d) & \stackrel{f}{\longrightarrow} & (X, d)
\end{array}
$$

and the chaotic relationships between $f$ and $\bar{f}$ has been exhaustively studied in the last years, where $(X, d)$ is a metric space, $f: X \rightarrow X$ is a continuous function, $\mathcal{K}(X)$ is the class of all non empty and compact subsets of $X, H$ is the Hausdorff metric on $\mathcal{K}(X)$ induced by $d$ and $\bar{f}$ is the natural extension of $f$ to $\mathcal{K}(X)$, i.e., $\bar{f}(A)=f(A)$ for all $A \in \mathcal{K}(X)$. In particular, some Devaney's connections in diagram (1) have been explored and, in this context, Román-Flores [7] showed that $\bar{f}$ transitive implies $f$ transitive and the converse implication is not necessarily true. Also, Román-Flores and Chalco-Cano [8] showed that $\bar{f}$ sensitive implies $f$ sensitive and, moreover, if $f$ has periodic density then $\bar{f}$ has periodic density. Later, Banks [2] shows that $\bar{f}$ transitive on $\mathcal{K}(X)$ is equivalent to $f$ weakly mixing on $X$.

Also, extending the above analysis, the next diagram

$$
\begin{array}{lll}
(\mathcal{F}(X), D) & \stackrel{\hat{f}}{\longrightarrow} & \underset{\uparrow}{\uparrow} \\
\uparrow & \mathcal{F}(X), D) \\
(X, d) & \stackrel{f}{\longrightarrow} & (X, d)
\end{array}
$$

and the chaotic relationships between $f$ and $\hat{f}$ has been also recently studied (see [10, 3, 6], where $\mathcal{F}(X)$ is the class of all non empty and compact fuzzy sets on $X, \hat{f}$ is the Zadeh's extension of $f$ to $\mathcal{F}(X)$ and $D$ is the extension of the Hausdorff metric to $\mathcal{F}(X)$ which is defined by

$$
D(u, v)=\sup _{\alpha \in[0,1]} H\left([u]^{\alpha},[v]^{\alpha}\right),
$$

where $[u]^{\alpha}$ is the $\alpha$-level of $u$ for $\alpha>0$ and $[u]^{0}$ is the support of $u$.

Other chaotic relations between $f, \bar{f}$ and $\hat{f}$ can be found in [9].

However in mathematical modelling, in the most of real applications it is necessary taking into account some additional considerations such that vagueness or uncertainty, which implies the use of interval and/or fuzzy-interval parameters and, consequently, to deal with interval and/or fuzzy-interval systems, that is to say, it is necessary to study of the following new diagrams:

$$
\begin{array}{lll}
\left(\mathcal{K}_{c}(X), H\right) & \stackrel{\bar{f}}{\longrightarrow} & \left(\mathcal{K}_{c}(X), H\right) \\
\uparrow & \uparrow \\
(X, d) \stackrel{f}{\longrightarrow} & (X, d)
\end{array}
$$

and

$$
\begin{array}{lll}
\left(\mathcal{F}_{c}(X), H\right) & \stackrel{\bar{f}}{\longrightarrow} & \left(\mathcal{F}_{c}(X), H\right) \\
\uparrow & \uparrow \\
(X, d) & \stackrel{f}{\longrightarrow} & (X, d)
\end{array}
$$

joint with the study of the connection between its respective dynamical relationships, and where $\mathcal{K}_{c}(X)$ and $\mathcal{F}_{c}(X)$ denotes the class of all non empty compact-convex subsets of $X$ and the class of all non empty compact-convex fuzzy sets on $X$, respectively.

In this direction, the following interesting example is analyzed by the authors in [7].

Example. Consider the "tent" function $f:[0,1] \rightarrow[0,1]$ defined by

$$
f(x)=\left\{\begin{array}{lll}
2 x & \text { if } \quad 0 \leq x \leq \frac{1}{2} \\
2(1-x) & \text { if } \quad \frac{1}{2} \leq x \leq 1
\end{array}\right.
$$

It is well known that $f$ is D-chaotic on $[0,1]$ (see [4]). Moreover, because $f$ is a mixing function on $[0,1]$ then $\bar{f}$ is transitive on $\mathcal{K}([0,1])$ (see[2]). Also, we observe that $x=\frac{2}{3}$ is a fixed point of $f$. On the other hand, it is clear that if $K$ is a compact and convex subset of $X=[0,1]$, then $\bar{f}(K)$ is also a compact and convex subset of $X$ and consequently, 
if we denote $\mathcal{K}_{c}([0,1])$ the class of all closed subintervals of $[0,1]$, then we can consider $\bar{f}$ as an application $\bar{f}: \mathcal{K}_{c}([0,1]) \rightarrow \mathcal{K}_{c}([0,1])$. We recall that $\mathcal{K}_{c}([0,1])$ is a closed subspace of $\mathcal{K}([0,1])$ in $H$-metric (see [5]).

Now, considering the open balls $B\left([0,1], \frac{1}{10}\right)$ and $B\left(\{0\}, \frac{1}{10}\right)$ in $\mathcal{K}_{c}([0,1])$, we have that

$$
K \in B\left([0,1], \frac{1}{10}\right) \Rightarrow \frac{2}{3} \in K
$$

and, consequently, $\frac{2}{3} \in \bar{f}^{p}(K), \forall p \in \mathbb{N}$. On the other hand, if $F \in B\left(\{0\}, \frac{1}{10}\right)$ then $F \subseteq[0,1 / 10]$, which implies that $H\left(\bar{f}^{p}(K), F\right) \geq \frac{17}{30}$ for every $K \in$ $B\left([0,1], \frac{1}{10}\right)$ and $F \in B\left(\{0\}, \frac{1}{10}\right)$.

Therefore,

$$
\bar{f}^{p}\left(B\left([0,1], \frac{1}{10}\right)\right) \bigcap B\left(\{0\}, \frac{1}{10}\right)=\emptyset, \forall p \in \mathbb{N},
$$

and, consequently, $\bar{f}$ is not transitive on $\mathcal{K}_{c}([0,1])$.

This example shows that a partial extension of $f$ (for instance, to the class $\mathcal{K}_{c}([0,1])$ ) can be not transitive, whereas the total extension of $f$ to $\mathcal{K}([0,1])$ is transitive.

Also, and in connection with above discussion, Kupka in [6] shows the following interesting relations between the transitivity of the fuzzy-interval extension $\hat{f}$ and the transitivity of the interval extension $\bar{f}$ :

Proposition 1. (Kupka [6, Prop. 2]) If $\hat{f}$ is transitive on $\mathcal{F}(X)$ then $\bar{f}$ is transitive on $\mathcal{K}(X)$.

Proposition 2. (Kupka [6, Prop. 3]) If $\hat{f}$ is transitive on $\mathcal{F}_{c}(X)$ then $\bar{f}$ is transitive on $\mathcal{K}_{c}(X)$,

Thus, the natural question that arises now is the following: what condition for an interval function $f:[a, b] \rightarrow[a, b]$ guarantees the transitivity of $\bar{f}$ and/or $\hat{f}$ on the interval and fuzzy-interval subextension $\mathcal{K}_{c}(X)$ and $\mathcal{F}_{c}(X)$, respectively?

The aim of this work is to show that, when $X=I$ is an interval, then $\bar{f}$ and $\hat{f}$ are not transitive, for all self continuous function $f$ defined on $[a, b]$.

\section{Main result}

In this section we will present our main results of this work.

Lemma $1 \bar{f}$ transitive on $\mathcal{K}_{c}([a, b])$ implies $f$ transitive on $[a, b]$.

Proof. Let $U, V$ nonempty open subsets of $X=[a, b]$. Thus, we can choose $x \in X, y \in Y$ and $\epsilon>0$ such that $B(x, \epsilon) \subset U$ and $B(y, \epsilon) \subset V$. Now, in $\mathcal{K}_{c}([a, b])$ consider the open balls $B(\{x\}, \epsilon)$ and $B(\{y\}, \epsilon)$ in $H$-metric. Then, due transitivity of $\bar{f}$ on $\mathcal{K}_{c}([a, b])$, there exists $n \in N$ such that $\bar{f}^{n}(B(\{x\}, \epsilon)) \cap B(\{y\}, \epsilon) \neq \emptyset$.

Therefore, there exists an interval $J \in$ $B(\{x\}, \epsilon)$ such that $\bar{f}^{n}(J)=f^{n}(J) \in B(\{y\}, \epsilon)$. But then $J \subset B(x, \epsilon)$ and, analogously, $f^{n}(J) \subset B(y, \epsilon)$, which implies that $f^{n}(B(x, \epsilon)) \cap B(y, \epsilon) \neq \emptyset$ and, consequenly, $f^{n}(U) \cap V \neq \emptyset$. That is, $f$ is a transitive function on $[a, b]$.

The following result shows that self continuous intervalar functions have no transitive intervalar extensions.

Theorem 1. Let $f:[a, b] \rightarrow[a, b]$ an arbitrary continuous function. Then $\bar{f}$ is not transitive on $\mathcal{K}_{c}([a, b])$.

Proof. By Schauder Theorem, $f$ has at least one fixed point $p \in[a, b]$.

Case 1) Suppose that $p \in(a, b)$ and let $r=\max \{p-$ $a, b-p\}$. Without loss of generality, we can suppose that $r=p-a$ and, because $a<b$, it is clear that $r>0$.

Now, let $r^{\prime}=b-p>0$ and let $\epsilon=\frac{r^{\prime}}{2}$. Then, if we consider the open balls $B([a, b], \epsilon), B(a, \epsilon) \in$ $\mathcal{K}_{c}([a, b])$, then it is clear that

$$
K \in B([a, b], \epsilon) \Rightarrow p \in K \Rightarrow p \in \bar{f}^{n}(K)
$$

for all $n \in \mathbb{N}$.

On the other hand,

$$
F \in B(a, \epsilon) \Rightarrow H(F, a)<\epsilon \rightarrow F \subseteq[a, a+\epsilon] .
$$

Therefore, because $r^{\prime}<r$ we have

$$
H\left(\bar{f}^{n}(K), F\right) \geq p-a-\epsilon=r-\frac{r^{\prime}}{2}>0
$$

$\forall K \in B([a, b], \epsilon), \forall F \in B(a, \epsilon), \forall n \in \mathbb{N}$.

Thus,

$$
\bar{f}^{n}(B([a, b], \epsilon)) \cap B(a, \epsilon)=\emptyset, \forall n \in \mathbb{N}
$$

and, consequentlty, in this case $\bar{f}$ is not transitive on $\mathcal{K}_{c}([a, b])$.

Case 2) Supposse that $f$ has no fixed points in $(a, b)$. Then, due to continuity of $f$, we have that $f(x)>x$ for all $x \in(a, b)$ or $f(x)<x$ for all $x \in(a, b)$. This clearly implies that $f$ is a not transitive function and consequently, due Lemma $1, \bar{f}$ is not transitive on $\mathcal{K}_{c}([a, b])$.

To finalize this sectin we will prove that self continuous interval functions have no transitive fuzzy-interval extensions.

Theorem 2. Let $f:[a, b] \rightarrow[a, b]$ an arbitrary continuous function. Then $\hat{f}$ is not transitive on $\mathcal{F}_{c}([a, b])$.

Proof. If we suppose that $\hat{f}$ is transitive on $\mathcal{F}_{c}([a, b])$ then, due to Proposition 3 , we have that 
$\bar{f}$ is transitive on $\mathcal{K}_{c}([a, b])$, in contradiction with Theorem 1.

Remark Let $I=[a, b]$ be a compact interval. If we denote by $\mathrm{TE}(\mathrm{I}, \mathrm{f})$ the topological entropy of the dynamical system (I,f), Cánovas \& Kupka in [3] shows the following interesting result

$$
T E([a, b], f)=T E\left(\mathcal{K}_{c}([a, b])\right)=T E\left(\mathcal{F}_{c}([a, b])\right) .
$$

This means that, in the interval case and from the point of view of the topological entropy, the complexity of the original system $(f, d)$, the set-system $(\bar{f}, H)$ and the fuzzy-system $(\hat{f}, D)$ are equal.

Thus, as we can see, this situation is very different from the transitive point of view where, in general, the complexity of the original system decreases strongly when we extend the original system to the interval and fuzzy interval context, which could be very useful in real applications when we want taking into account the incertainty involved in the most of dynamical systems.

\section{Aknowledgement}

This work was supported by Conicyt-Chile through Projects Fondecyt 1120674,1151159 and 1151154.

\section{References}

[1] J. Banks, J. Brooks, G. Cairns, G. Davis and P. Stacey, On the Devaney's definition of chaos, Amer.Math.Month., 99:332-334, AMS,1992.

[2] J. Banks. Chaos for induced hyperspace maps. Chaos, Solitonser Fractals, 25:681-685, Elsevier, 2005.

[3] José J. Cánovas and J. Kupka, On the topological entropy on the space of fuzzy numbers, Fuzzy Sets and Systems, 257:132-145, Elsevier, 2014.

[4] R.L. Devaney. A first course in chaotic dynamical systems: Theory and Experiments, Perseus Books, Boston, 1992.

[5] Klein E. and Thompson A. Theory of correspondences, Wiley-Interscience, New York, 1984.

[6] J. Kupka, On Devaney chaotic induced fuzzy and set-valued dynamical systems, Fuzzy Sets and Systems, 177:34-44, Elsevier, 2011.

[7] H. Román-Flores, A note on transitivity in setvalued discrete systems. Chaos, Solitons\& Fractals, 17:99-104, Elsevier, 2003.

[8] H. Román-Flores and Y. Chalco-Cano, Robinson's chaos in set-valued discrete systems, Chaos, Solitons\& Fractals, 25:3542, Elsevier, 2005.

[9] H. Román-Flores, Y. Chalco-Cano, G.N. Silva and Jiří Kupka, On turbulent, erratic and other dynamical properties of Zadeh's extensions, Chaos, Solitons $\&$ Fractals, 44:990-994, Elsevier, 2011.

[10] H. Román-Flores and Y. Chalco-Cano Some chaotic properties of Zadeh's extensions, Chaos, Solitons\& Fractals, 35:452-459, Elsevier, 2008.
[11] M. Vellekoop and R.Berglund, On intervals, Transitivity $=$ Chaos, Amer.Math.Month., 101:353-355, AMS, 1994. 